\title{
Including service users in trials and rigorous studies in health and social care: developing a standard operating procedure for researchers
}

\author{
Bridie Angela Evans ${ }^{1 *}$, Emma Bedson², Philip Bell ${ }^{3}$, Hayley Hutchings ${ }^{1}$, Lesley Lowes ${ }^{4}$, David Rea ${ }^{5}$, Anne Seagrove ${ }^{1}$, \\ Stefan Siebert ${ }^{1}$, Graham Smith ${ }^{3}$, Helen Snooks ${ }^{1}$, Marie Thomas ${ }^{6}$, Kym Thorne ${ }^{1}$, Ian Russell ${ }^{1}$
}

From Clinical Trials Methodology Conference 2011

Bristol, UK. 4-5 October 2011

\section{Background}

Involving service users in research is encouraged as a way to improve research quality, relevance and accountability and is a pre-requisite for many funding bodies. Existing guidance for researchers on how to do this mainly discusses general principles. Some researchers may question the value, feasibility and impact of including service users and limit the scope of involvement. We defined service users as patients, carers, people eligible for a service or anyone relevant to the trial inclusion criteria.

\section{Objective}

To develop a standard operating procedure (SOP) to give researchers detailed guidance on how to include service users in trials and other rigorous studies.

\section{Methods}

Researchers with experience of service user inclusion and service users currently involved in trials adopted by the West Wales Organisation for Rigorous Trials in Health (WWORTH), the Clinical Trials Unit based in Swansea University, collaborated to develop the SOP for service user inclusion.

\section{Results}

We articulated core principles of equality underpinning the SOP and guidance on how to achieve these. Processes to recruit and engage service users were set out. We developed a framework for inclusion in research which defined minimum core levels of collaboration

${ }^{1}$ College of Medicine, Swansea University, Swansea, SA2 8PP, UK Full list of author information is available at the end of the article plus additional consultation and collaboration opportunities. A flow diagram identified when and how to include service users at each of the Medical Research Council's five stages of developing and evaluating complex interventions in health [1]. We listed people across the research team responsible for including service users in studies and promoting an inclusion culture and highlighted importance of training for researchers and service users. We stated that service users should be included as early as possible in the research process with a minimum of two on all formal trial groups and committees. We proposed a minimum $1 \%$ of total research budget should be set aside to include service users and sufficient additional time built in to allow for full inclusion.

\section{Conclusion}

Supporting good practice when including service users in research could benefit the relevance, accountability and quality of health and social care research. This SOP provides guidance to researchers to successfully involve service users in developing proposals, undertaking rigorous research and creating a culture of routine service user inclusion in research at all stages. The UK Clinical Research Collaboration should require trials to demonstrate service user inclusion and research funders must set aside sufficient funds and time for this in research proposals.

\section{Acknowledgements}

We are grateful to the service users and researchers who contributed to this Standard Operating Procedure.

This study is presented on behalf of the West Wales Organisation for Rigorous Trials in Health (WWORTH). 


\section{Author details}

${ }^{1}$ College of Medicine, Swansea University, Swansea, SA2 8PP, UK. ${ }^{2}$ North

Wales Organisation for Randomised Trials in Health (NWORTH), Bangor University, Bangor, LL57 2DG, UK. ${ }^{3}$ Service User, Cardiff University, CF14 4XN, UK. ${ }^{4}$ School of Nursing and Midwifery Studies, Cardiff University, CF14 4XN,

UK. ${ }^{5}$ College of Human and Health Sciences, Swansea University, Swansea, SA2 8PP, UK. 'School of Education, University of Wales Newport, Newport, NP18 3QT, UK.

Published: 13 December 2011

\section{Reference}

1. Craig P, et al: Developing and evaluating complex interventions: the new Medical Research Council guidance. British Medical Journal 2008, 337:979-983.

doi:10.1186/1745-6215-12-S1-A83

Cite this article as: Evans et al:: Including service users in trials and rigorous studies in health and social care: developing a standard operating procedure for researchers. Trials 2011 12(Suppl 1):A83.

\section{Submit your next manuscript to BioMed Central} and take full advantage of:

- Convenient online submission

- Thorough peer review

- No space constraints or color figure charges

- Immediate publication on acceptance

- Inclusion in PubMed, CAS, Scopus and Google Scholar

- Research which is freely available for redistribution

Submit your manuscript at www.biomedcentral.com/submit 